\title{
FATORES DE RISCO PARA O DESENVOLVIMENTO DE DISTÚRBIOS ALIMENTARES: UM ESTUDO EM UNIVERSITÁRIAS
}

\author{
RISK FACTORS IN THE DEVELOPMENT OF \\ EATING DISORDERS: STUDY IN A GROUP OF COLEGE WOMEN
}

\author{
Giovanna Medeiros Rataichesck FIATES' \\ Raquel Kuerten de SALLES ${ }^{1}$
}

\section{RESUMO}

Este trabalho buscou identificar, em mulheres universitárias, aquelas que apresentavam fatores de risco para o desenvolvimento de distúrbios alimentares. Aplicou-se o questionário Eating Attitudes Test em 221 mulheres (114 estudantes de nutrição e 107 estudantes de outras áreas não relacionadas à saúde). Os resultados indicaram que 22,17\% das estudantes apresentaram fatores de risco para o desenvolvimento de distúrbio alimentar, sendo que no grupo de alunas da nutrição o percentual foi maior (25,43\%) do que no grupo de estudantes de outros cursos (18,69\%). Embora a diferença não tenha sido significativa, as futuras nutricionistas podem estar inseridas em um ambiente mais favorável ao desenvolvimento de distúrbios alimentares.

Termos de indexação: distúrbios alimentares, anorexia, estudantes, fatores de risco.

\begin{abstract}
This work studied a group of 221 college women, to identify those who presented risk factors compatible with the development of eating disorders. The Eating Attitudes Test was applied to 221 women (114 nutrition students and 107 students from other areas, unrelated to the health field). Results indicated that $22.17 \%$ of the group presented risk factors in the development of an eating disorder. Nutrition students presented a higher percentage (25.43\%) than the other students (18.69\%), but this difference was not significant. However, women who study nutrition may be exposed to an environment more propitious to the development of eating disorders.
\end{abstract}

Index terms: eating disorders, anorexia, students, risk factors.

\section{INTRODUÇÃO}

Os distúrbios alimentares ocorrem mais freqüentemente no sexo feminino, representando $95 \%$ dos casos. Estudos têm demonstrado um aumento da incidência nas últimas duas décadas (Castro \& Goldstein, 1995). O quadro costuma ter como fator desencadeante algum evento significativo como perdas, separações, mudanças, doenças orgânicas, distúrbios da imagem corporal, depressão, ansiedade e, até mesmo, traumas de infância,

\footnotetext{
(1) Departamento de Nutrição, Centro de Ciências da Saúde, Universidade Federal de Santa Catarina. Campus Universitário, Trindade, 88010-970, Florianópolis, SC, Brasil. Correspondência para/Correspondence to: G.M.R. FIATES.
} 
como abuso sexual. No entanto, a forma como estes fatores vão atuar como causa do distúrbio ainda não está esclarecida (Paxton, 1998).

A anorexia nervosa é caracterizada por uma restrição alimentar auto imposta com seqüelas graves, características obsessivo-compulsivas e crenças irracionais que freqüentemente complicam o tratamento. A instalação da doença de forma crônica provoca a princípio desnutrição e desidratação. Apesar dos pacientes negarem fome, são comuns as queixas de fadiga, fraqueza, tonturas e visão turva. As complicações tornam-se graves uma vez que são utilizados métodos drásticos para se conseguir a perda cada vez maior de peso corporal (Wiseman et al., 1988).

O sexo feminino de uma maneira geral é muito vulnerável à aceitação das pressões sociais, econômicas e culturais associadas aos padrões estéticos. A sociedade rejeita, discrimina e reprova pessoas obesas. Perante esta situação muitas mulheres encontram-se insatisfeitas com seu corpo. O medo da obesidade faz com que um número cada vez maior de mulheres faça dieta, controle neuroticamente o peso corporal, exercite-se de maneira exaustiva e faça uso de laxantes, diuréticos e drogas anorexígenas (Beatty \& Finn, 1995).

Nas sociedades afluentes, ao mesmo tempo em que observamos uma oferta abundante de alimentos de alto teor energético e de rápido consumo, a vida cotidiana é cada vez mais sedentária, tornando mais penosa a tarefa de manter o corpo magro. Modelos e atrizes de sucesso, representantes dos padrões ideais de beleza feminina, são extremamente magras e muitas vezes apresentam um corpo de pré-adolescente com formas pouco definidas (Nunes, 1998).

Assim como os fatores sociais, culturais e psicológicos estão envolvidos na gênese da anorexia nervosa, diferenças na percepção da fome e da saciedade parecem ser a expressão de mecanismos fisiológicos envolvidos na regulação do comportamento alimentar (Garfinkel et al., 1987).

O tratamento dos transtornos alimentares sofreu avanços nas últimas décadas. Hoje, sabe-se que os melhores resultados são alcançados através de equipes multiprofissionais (Whisenant \& Smith, 1995), e que é fundamental trabalhar no campo da prevenção, identificando os indivíduos em risco (Piran, 1997).
O objetivo do trabalho foi avaliar a presença de fatores de risco para a ocorrência de distúrbios alimentares em mulheres universitárias.

\section{CASUÍSTICA E MÉTODOS}

Um dos métodos utilizados para avaliar a presença de fatores de risco para distúrbios é o questionário Eating Attitudes Test (EAT), instrumento que contém 26 perguntas sobre comportamento alimentar e imagem corporal (Garner \& Garfinkel, 1979).

Aplicou-se o questionário EAT nas alunas da Universidade Federal de Santa Catarina, matriculadas no curso de Nutrição que aceitaram responder o questionário ( $\mathrm{n}=114)$; e em um número similar de estudantes matriculadas em cursos desvinculados da área da Saúde $(n=107)$. A faixa etária da população estudada foi de 19 a 25 anos de idade.

Foram coletados também, dados de peso e altura referidos, para posterior cálculo do Ìndice de Massa Corporal (IMC). A classificação do IMC foi feita de acordo com World Health... (1998).

O questionário EAT foi considerado indicador de risco para o desenvolvimento de um distúrbio nutricional, quando o número de respostas positivas foi igual ou superior a 21 (Garner \& Garfinkel, 1979). Paralelamente, foram analisadas as respostas citadas com maior freqüência nos questionários EAT positivos (EAT+).

Os testes estatísticos foram feitos através do programa Epi Info versão 6, aplicou-se o teste quiquadrado e odds ratio.

\section{RESULTADOS}

Do total de estudantes ( $\mathrm{n}=221), 22,17 \%$ foram classificadas como EAT+ (Tabela 1). Em um trabalho semelhante, Castro \& Goldstein (1995) encontraram um percentual de 20\%; Pastore et al. (1996) encontraram $15 \%$, e consideram este o máximo esperado para este grupo populacional.

No grupo de estudantes de nutrição, o percentual de estudantes EAT + $(25,43 \%)$ foi maior do que no grupo

Tabela 1. Resultados da aplicação do questionário EAT em estudantes universitárias.

\begin{tabular}{|c|c|c|c|c|c|c|c|c|c|}
\hline \multirow{3}{*}{ Area de estudo } & \multirow{3}{*}{ Questionáriosaplicados } & & & \multicolumn{6}{|c|}{ Estado nutricional das estudantes EAT $+(n=49)$} \\
\hline & & \multicolumn{2}{|c|}{ EAT+ } & \multicolumn{2}{|c|}{ Eutrofia } & \multicolumn{2}{|c|}{ Magreza } & \multicolumn{2}{|c|}{ Sobrepeso } \\
\hline & & $\mathrm{n}$ & $\%$ & $\mathrm{n}$ & $\%$ & $\mathrm{n}$ & $\%$ & $\mathrm{n}$ & $\%$ \\
\hline Nutrição & 114 & 29 & 25,43 & 25 & & 1 & & 3 & \\
\hline Outros cursos & 107 & 20 & 18,69 & 17 & & 3 & & 0 & \\
\hline Total & 221 & 49 & 22,17 & 42 & 85,70 & 4 & 8,16 & 3 & 6,12 \\
\hline
\end{tabular}


Tabela 2. Respostas mais freqüentes nos questionários de estudantes EAT + do curso de nutrição e de cursos desvinculados da área da saúde.

\begin{tabular}{|c|c|c|c|c|}
\hline \multirow{2}{*}{ Respostas } & \multicolumn{2}{|c|}{ EAT + outros cursos $(n=20)$} & \multicolumn{2}{|c|}{ EAT + nutrição $(\mathrm{n}=29)$} \\
\hline & $\mathrm{n}$ & $\%$ & $\mathrm{n}$ & $\%$ \\
\hline Hábito de fazer dieta & 8 & 28,0 & 4 & 20,0 \\
\hline Uso de produtos dietéticos & 10 & 34,5 & 7 & 35,0 \\
\hline Desejam ser mais magras & 14 & 48,3 & 11 & 55,0 \\
\hline Referem que a comida controla suas vidas & 9 & 31,0 & 4 & 20,0 \\
\hline Fazem exercício para queimar calorias & 21 & 72,4 & 15 & 75,0 \\
\hline
\end{tabular}

de outros cursos $(18,69 \%)$ entretanto, a diferença não foi significativa. Observou-se ainda que o número de casos de magreza foi maior no grupo de outros cursos, sendo o sobrepeso mais prevalente nas estudantes de nutrição.

Poucas pesquisas sobre alterações nutricionais com universitários têm sido realizadas no Brasil, mas trabalhos realizados em outros países mostram que este grupo pode estar sujeito a carências ou excessos nutricionais (Hendricks \& Herbold, 1998).

Além da avaliação do somatório de pontos do questionário EAT, foram também tabuladas as respostas positivas encontradas com maior freqüência (Tabela 2). O desejo de ser mais magra e a atividade física exaustiva com objetivo de perder peso foram as respostas mais freqüentes entre os 2 grupos.

Fatores como descontentamento com a imagem corporal, preocupação com o peso e formas de perder peso nocivas à saúde são encontrados, mesmo em estudantes com o peso adequado (Schwitzer et al., 1998). Estudos com universitários demonstraram que estes podem comprometer seu estado nutricional devido a alimentação inadequada por modismos dietéticos, omissão de refeições, consumo de fast-foods e rejeição a certos grupos de alimentos (Fennell, 1997).

\section{DISCUSSÃO}

O percentual de mulheres com possibilidade de desenvolver um distúrbio alimentar foi maior no grupo de estudantes de nutrição do que no grupo formado por estudantes de outros cursos. Também no grupo de estudantes de nutrição foi maior o número de mulheres com sobrepeso.

A literatura refere maior incidência de distúrbios alimentares em alguns grupos ocupacionais: modelos, atrizes, atletas e nutricionistas parecem estar particularmente mais vulneráveis ao desenvolvimento de transtornos alimentares, seja pelo consumo alimentar deficiente ou excessivo (Nunes, 1998). Além destes fatores, podemos inferir que pessoas já preocupadas com seu peso (adequado ou não) e imagem corporal, optem por esta área de estudo, justamente por já terem um interesse pessoal pelo tema.

Encontramos uma preocupação excessiva com o consumo de produtos dietéticos, e o hábito freqüente de fazer dieta, comportamentos característicos de indivíduos suscetíveis aos distúrbios alimentares. Estes apresentam preferências alimentares e atitudes em relação aos alimentos, que os leva a ingerir somente alimentos considerados "seguros" (Sunday \& Halmi, 1997).

Outras características observadas com freqüência nos questionários foram: o desejo de ser mais magra, intolerância ao excesso de peso e preocupação obssessiva com a comida. O medo mórbido de engordar, o constante desejo de perder peso e a distorção da imagem corporal são sinais evidentes dos transtornos alimentares, e estão altamente relacionados com o seu desenvolvimento (Gidwani \& Rome, 1997; Walsh \& Devlin, 1998).

Muitas vezes a busca pela magreza não requer apenas a seleção criteriosa dos alimentos a serem ingeridos; é preciso ainda associar o exercício físico para a obtenção do objetivo tão desejado. Neste aspecto, o hábito de freqüentar academias ou fazer caminhadas diárias pode estar desvinculado dos efeitos benéficos dos exercícios físicos sobre a saúde. A prática de atividade física é feita exclusivamente para resultar em perda de peso. Este fato foi observado nos dois grupos estudados, onde mais de $70 \%$ das estudantes EAT + relataram exercitar-se apenas com o propósito de queimar energia.

\section{CONCLUSÃO}

O percentual encontrado, de mais de $20 \%$ de estudantes com EAT+, foi considerado preocupante, evidenciando a tendência atual de aumento na prevalência de distúrbios alimentares.

As futuras nutricionistas estão em contato constante com o alimento; sabem que "boa aparência" pode ser uma importante medida de valor pessoal rumo a uma profissão de sucesso. Além disso, possuem conhecimentos quantitativos e qualitativos a respeito dos alimentos que praticamente as obrigam a manterem-se de acordo com os rígidos padrões estéticos vigentes. Estes fatores podem sugerir que as estudantes de nutrição estão inseridas em um ambiente mais favorável ao desenvolvimento de distúrbios nutricionais.

No entanto, não podemos esquecer que a etiologia dos distúrbios alimentares é multidimensional e que 
inúmeros outros fatores parecem mediar o impacto da cultura, cuja participação parece indiscutível, no comportamento individual. Torna-se imprescindível que as investigações continuem no sentido de se conhecer melhor as causas que conduzem ao surgimento de distúrbios alimentares e sua ligação com os padrões culturais atuais.

\section{AGRADECIMENTOS}

Às acadêmicas do curso de Graduação em Nutrição, Alessandra Erdmann, Csele Vand Sand e Heveny Capello, pelo valoroso auxílio na execução da pesquisa.

\section{REFERÊNCIAS BIBLIOGRÁFICAS}

BEATTY, D., FINN, S.C. Position of the American Dietetic Association and the Canadian Dietetic Association: women's health and nutrition. Journal of the American Dietetic Association, Chicago, v.95, n.3, p.362-366, 1995.

CASTRO, M.J., GOLDSTEIN, J.S. Eating attitudes and behaviors of pre-and postpubertal females: clues to the etiology of eating disorders. Physiology and Behavior, Elmsford, v.58, n.1, p.15-23, 1995.

FENNELL, R. Health behaviors of students attending historically black colleges and universities: results from the National College Health Risk Behavior survey. Journal of the American College Health, Washington DC, v.46, n.3, p.109-117, 1997.

GARFINKEL, P.E., GARNER, D.M., GOLDBLOOM, D.S. Eating disorders: implications for the 1990's. Canadian Journal of Psychiatry, Ottawa, v.7, n.32, p.624-631, 1987.

GARNER, D.N., GARFINKEL, P.E. The eating attitudes test: an index of the symptoms of anorexia nervosa. Psychological Medicine, v.2, n.9, p.273-279, 1979.

GIDWANI, P.G., ROME, S.E. Eating disorders. Clinical Obstetrics and Gynecology, Philadelphia, v.40, n.3, p.601-615, 1997.
HENDRICKS, K.M., HERBOLD, N.H. Diet, activity and other health related behaviors in college-age women. Nutrition Reviews, New York, v.56, n.3, p.65-75, 1998.

NUNES, M.A.A. Transtornos alimentares e obesidade. Porto Alegre : Artes Médicas Sul, 1998. 215p.

PASTORE, D.R., FISHER, M., FRIEDMAN, S.B. Abnormalities in weight status, eating attitudes and eating behaviors among urban high school students. Journal of Adolescent Health, New York, v.18, n.5, p. 312-319, 1996.

PAXTON, S.J. Current issues in eating disorders research. Journal of Psychosomatic Research, Oxford, v.44, n.34, p.297-299, 1998.

PIRAN, N. Prevention of eating disorders: directions for future research. Psychopharmacology Bulletin, Rockville, v.33, n.3, p.419-423, 1997.

SCHWITZER, A.M., BERGHOLZ, K., DORE,T. et al. Eating disorders among college women: prevention, education and treatment responses. Journal of the American College Health, Washington DC, v.46, n.5, p.199-207, 1998.

SUNDAY, R.S., HALMI, A.C. Eating behavior and eating disorders: the interface between clinical research and clinical practice. Psychopharmacology Bulletin, Rockville, v.33, n.3, p.373-379, 1997.

WALSH, T.B., DEVLIN, J.M. Eating disorders: progress and problems. Science, Washington DC, v.280, n.5368, p.138790, 1998.

WHISENANT, S.L., SMITH, B.A. Eating disorders: current nutrition therapy and perceived needs in dietetics education and research. Journal of the American Dietetic Association, Chicago, v.95, n.10, p.1109-1112, 1995.

WORLD HEALTH ORGANIZATION. Obesity: preventing and managing the global epidemic. Geneva, 1998. 276p.

WISEMAN, C.V., HARRIS, W.A., HALMI, K.A. Eating disorders. Medical Clinics of North America, Philadelphia, v.82, n.1, p.145-159, 1988.

Recebido para publicação em 4 de julho e aceito em 7 de novembro de 2000 . 